\title{
Selection of Molding Method for CFRP Automotive Body Parts \\ - Resin injection vs. compression -
}

\author{
Yuji Kageyama 1*, Kenju Akai², Nariaki Nishino², Kazuro Kageyama² \\ 1 Kanazawa Institute of Technology \\ y_kageyama@neptune.kanazawa-it.ac.jp \\ 2 The University of Tokyo \\ akaikenju@gmail.com, Nishino, @tmi.t.u-tokyo.ac.jp, kageya- \\ malgiso.t.u-tokyo.ac.jp
}

\begin{abstract}
The carbon fiber reinforced plastic (CFRP) is now drawing attention in the automotive industry and has also recently been adopted for manufacturing bodies of mass produced electric vehicles. The CFRP molding method for automobile body can be largely categorized into the injection and compression methods. The essential difference between the two lies in whether or not the process involves an intermediary material and it became evident that this had a significant impact on quality and productivity. An evaluation was performed to determine which of these methods the valid method for basic molding, is by comparing the nature of such intermediary material with main components of automobile body, which led to the discovery of fundamental issues. A review on how technical developments thus far were considered with regards to such issues, it became evident that while significant progress was being made there was still a divergence from the expected target.
\end{abstract}

Keywords: Carbon Fiber Reinforced Plastic(CFRP), automobile industry, molding method

\section{Trends in Automobile Body Components and Expectations for CFRP1)}

A consideration of the transition of the materials used for automobiles indicates that the use of steel is on a gradual decline, while the use of lightweight materials, such as aluminum and resin, is starting to increase. The same is true for structural materials, with the use of pillars made from high-tension steel and aluminum for outer panels on the increase. CFRP, on the other hand, continues to be popular in the field of motor sports, such as F1 racing, since its use has been attributed to the sharp drop in the number of accident-related deaths of drivers. There has been an increase in the number of instances of its use for the structural components of supercars. It has been difficult to adopt this material for mass-produced automobiles, however, due to reasons such as its high cost. CFRP has, however, recently been seen in environmentally friendly vehicles, such as in the body of the BMW i3 and the stack frame of the Toyota Mirai. The material not only improves performance such as driving stability through weight reduction, but it is also expected to provide a means of reducing negative legacies, such as environmental problems.

\section{CFRP Molding Methods Currently Applied to Automobile Bodies}

adfa, p. 1, 2011.

(C) Springer-Verlag Berlin Heidelberg 2011 
Although there are great expectations for CFRP as a material for automobiles, its status has not yet been firmly established due to issues with cost and productivity. There has, however, been an overall reduction in cost. Carbon fiber manufacturers, furthermore, have set their sights on the development of carbon fiber with a higher number of filaments in the strands, not only for aerospace but also for automotive applications. Researchers have developed innovative varieties of carbon fiber specifically for automobiles, without the constraints of conventional carbon fiber manufacturing techniques, and further development is expected. In terms of productivity, on the other hand, a salient issue has been the time-consuming detachment of the product from the autoclave mold. This is the final step after the cutting, pasting, and layering of dozens of resin-soaked carbon fiber sheets in the mold, which are then sintered in an autoclave. Resin transfer molding (RTM), which is a resin injection method and carbon fiber sheet molding compound (C-SMC), which is a press method, have been adopted as innovative construction methods for the Lexus LFA sports car ${ }^{1)}$. Highpressure RTM (HP-RTM) molding has furthermore been implemented as an improvement over RTM and adopted for the engine hood and roof of the Lexus RCF. The construction method that used to be called structural reaction injection molding (S-RIM) formed the basis for the development of HP-RTM. Molding that can be completed within three minutes is now a reality, due to the improvement of equipment and the development of rapid curing resins. The fact that the body of the i3 model from BMW, which is a mass-produced electric vehicle, uses this construction method is truly ground-breaking. This will certainly be a trend to follow in the future. The current situation is such that a variety of other CFRP molding methods for automobiles are being researched, developed, and proposed. Research into CFRP using thermoplastic resins has recently been attracting considerable attention, with particular emphasis on special molding methods such as the thermoplastic CFRP sheet compression method.

\section{Analysis of CFRP Molding Methods for Automobile Bodies}

While it is obviously good that the industry is proposing a variety of CFRP molding methods for automobiles, not all molding manufacturers can quickly set up their equipment to start their research and begin mass production. All of these molding methods require special molding facilities which are not readily available and are, of course, quite expensive. In addition, material manufacturers often comment that, while they are interested in researching CFRP as a material for the future, they are at loss regards determining the molding method on which they should concentrate. Even if they were to concentrate on one particular molding method, they still would not know which components should be targeted, to whom they should offer their materials, or with whom they could consult in their efforts.

\section{1 Two molding methods}


We considered the two CFRP molding methods that are currently gaining the greatest attention for application to automobile bodies. Our findings are listed in Table 1. In many cases, the molding methods could be largely categorized into two types, namely, the injection type and the compression type. The injection type is often used to create relatively large components such as body frames or outer panels and includes methods such as RTM molding. This type of molding method has been applied to mass-produced vehicles such as the 13 from BMW, through the adoption of the HP-RTM molding method. This is a wet method that involves impregnating the carbon fiber base material (that has been formed into the shape of the intended product) with liquid resin. This is then composited and reactively cured with heat or the like. The compression method, on the other hand, is a dry method. The carbon fiber base materials are impregnated with resin in advance. The intermediary material, which has been half-cured or solidified, is made to flow under high pressure. It is in this aspect that the method differs from the injection method. These methods have actually been adopted for secondary frame components or outer panels made of CSMC or trunk lids made by prepreg compression molding (PCM), as was adopted for the Nissan GT-R. The stack frame of the Toyota Mirai, which is a fuel cell vehicle, is molded using a compression method, using a thermoplastic resin CFRP intermediary material, which has drawn considerable attention in recent years. The fundamental difference between these two methods is in whether they use an intermediary material, which has been prepared by compositing the resins and fibers in advance. These methods can be broadly categorized into the compression method that uses an intermediary material and the injection method that does not use any such material, with materials being composited within a mold, instead.

\section{2 Differences between Two Molding Methods}

The fundamental difference between these two methods is whether an intermediary material is used for preparing the composite material. Next, we will consider how this difference affects the level of quality or productivity (Fig.1).

An advantage of using an intermediary material is that the number of compositing processes at the manufacturing site is reduced by one. This leads to the shortening of the molding cycle, which better enables mass production. Conversely, the stability of

Table 1: CFRP molding method for automobile body

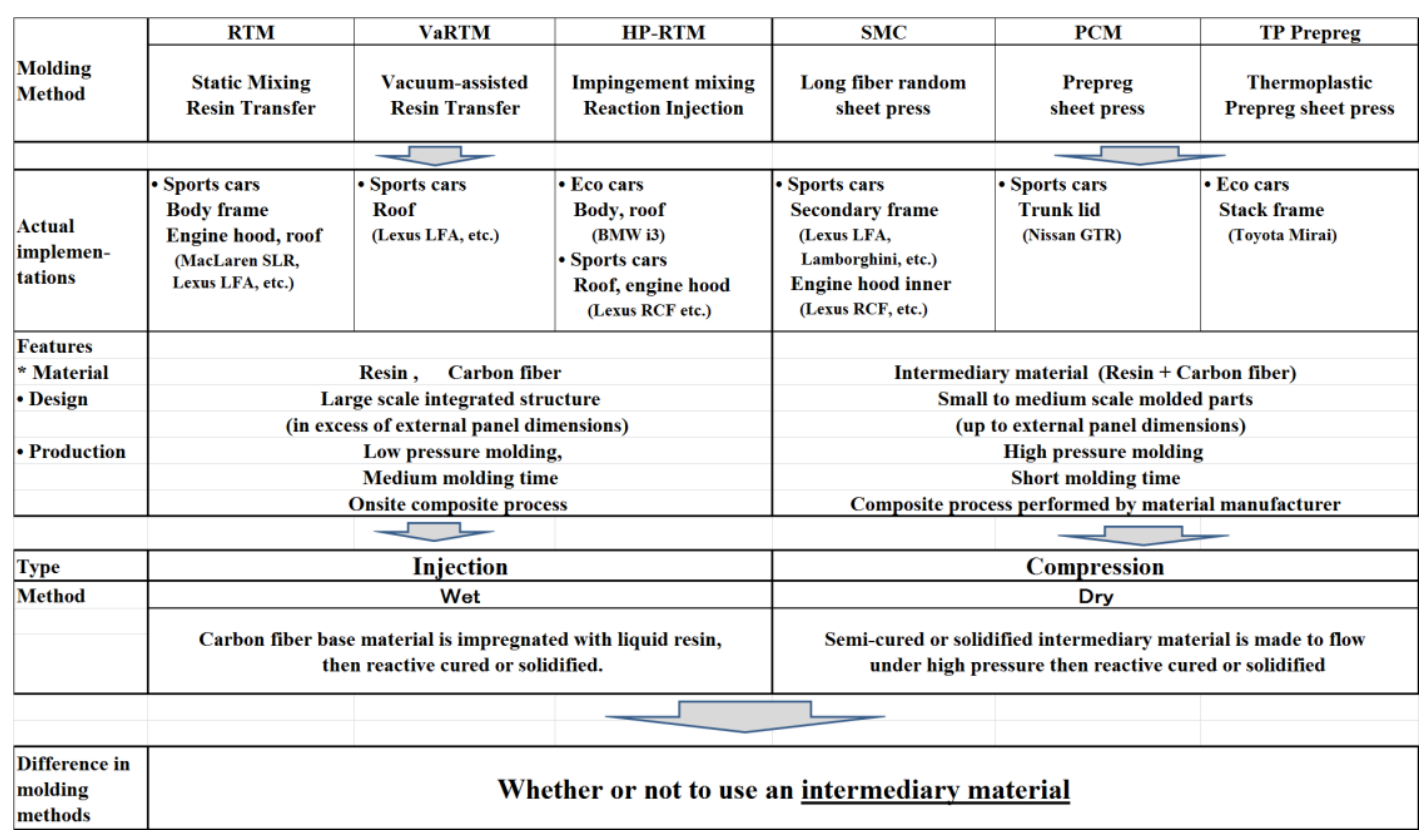




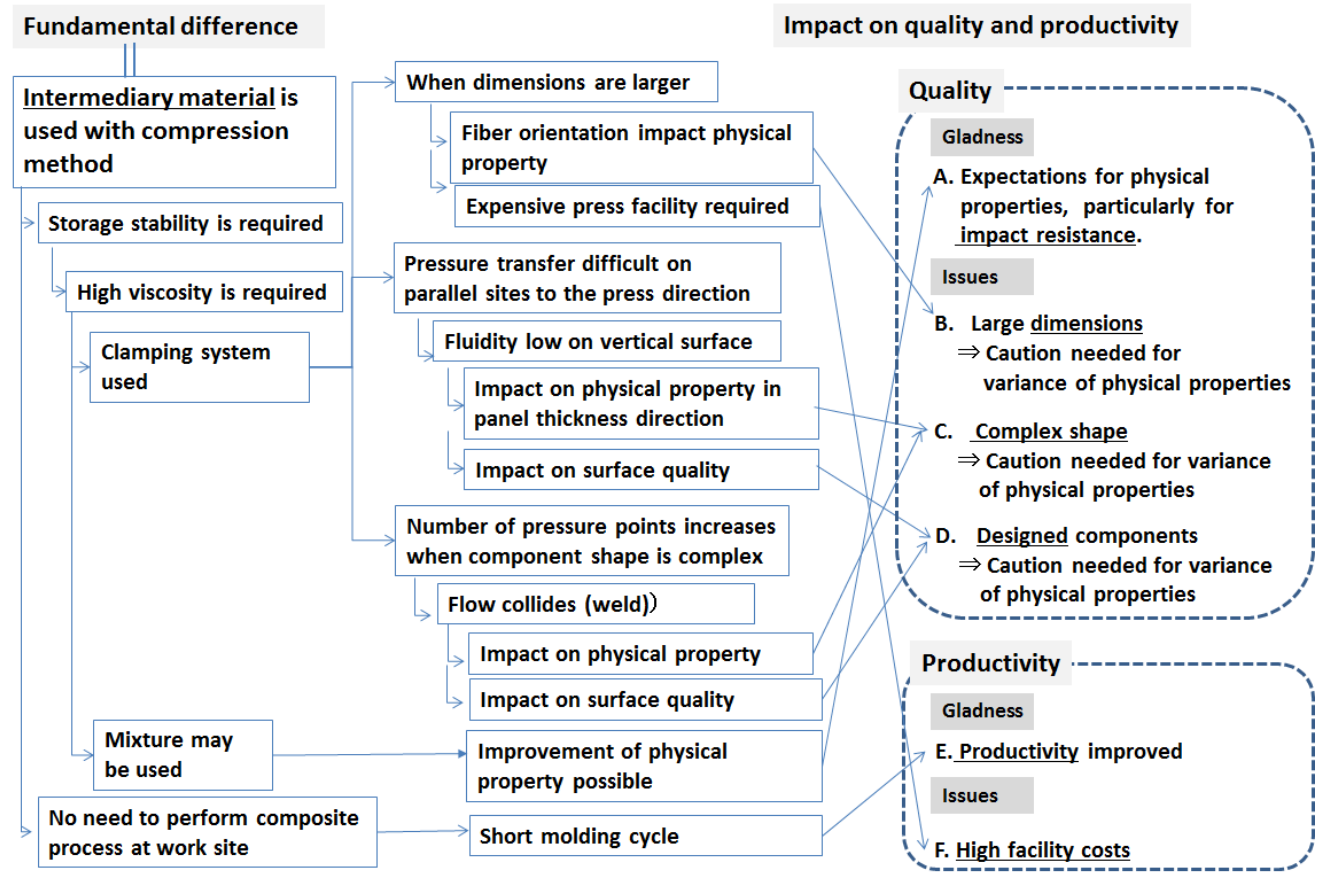

Fig.1. Impact from difference between injection and compression molding methods on quality and productivity

the intermediary material during storage presents an issue, since it incorporates unreacted resin. There are, of course, a number of aspects with regards to stability, but the most important of all relates to the prevention of resin outflow from an intermediary material in the storage environment. A raised level of viscosity is therefore required of the resin. Increasing the viscosity has a significant impact on quality and productivity. First of all, to mold a material into the shape of the product by using a mold, it is necessary to apply pressure to the intermediary material. This is provided by a pressbased clamping system. When the projected area of the product becomes large in such a case, even higher pressures becomes necessary to make the intermediary material flow. Depending on the product dimensions, very expensive press facilities may be necessary. Furthermore, fiber can easily be oriented in the flow direction and the variance in the physical properties can become significant, potentially having an impact on the surface quality of the product. The nature of this method is such that the pressure is less likely to be applied to the product sections in the direction of the mold clamping, which tends to adversely affect the fluidity of the intermediary material. The deterioration in the fluidity of the material has an impact on the physical properties and surface quality. When the shapes of the components are complex, the physical properties and surface quality of the product can vary. Furthermore, when the component shape is complex, the number of pressurizing points must increase, which leads to an increased number of locations where the flows caused by pressurization 
run into each other. This is more of an issue when the viscosity is high, such that the flows are unable to mix, which can have a significant impact on the physical properties and surface quality. Resin, however, is quite good at mixing, and thus can be used as a filler for shock absorption and for filling other functional materials. Taking advantage of this aspect, it would be possible to predict that, while the intermediary material is less likely to flow, the physical properties can be improved. This would be particularly advantageous for parts that need to be collision resistant.

There are advantages related to mass productivity and the respective physical properties attainable with the compression method which features the use of an intermediary material. However, caution is required in such aspects as the dimensions of the product, the complexity of the shape, or the design properties. The injection method offers the opposite properties.

\subsection{Main Components of Automobile Body and Two Molding Methods}

The properties of the two molding methods described above and the required characteristics of the main components of an automobile body were compared to evaluate their compatibility. The results are listed in Table 2 . The components were categorized into the body frame, lids that are opened and closed, reinforcements, as well as interior and exterior components. Parts were selected from these categories. In terms of productivity, furthermore, the assumption was that the components were mass produced for automobiles that are built at a rate of 10,000 units per month, in order to

Table 2: Fundamental molding method and main issues from aspects of "respective automobile components"

\begin{tabular}{|c|c|c|c|c|c|c|}
\hline \multirow{3}{*}{ 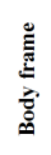 } & Floor panel & Large & Complex & None & Medium & Large \\
\hline & Sub-frame & Medium & Simple & None & Large & Large \\
\hline & Side sill & Medium & Simple & None & Large & Large \\
\hline \multirow{4}{*}{$\frac{n}{a}$} & Engine hood -Outer & Medium & $\begin{array}{c}\text { Simple } \\
\text { Complex }\end{array}$ & $\begin{array}{c}\text { Avail- } \\
\text { able }\end{array}$ & Small & Large \\
\hline & - Inner & Medium & Simple & None & Medium & Large \\
\hline & Back door -Outer & Medium & $\begin{array}{c}\text { Simple } \\
\text { Complex }\end{array}$ & $\begin{array}{c}\text { Avail- } \\
\text { able }\end{array}$ & Medium & Large \\
\hline & - Inner & Medium & Simple & None & Large & Large \\
\hline 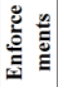 & Engine mount, etc. & Small & Simple & None & Medium & Large \\
\hline \multirow{2}{*}{ 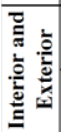 } & Rear spoiler & Small & Simple & $\begin{array}{c}\text { Avail- } \\
\text { able }\end{array}$ & Small & Large \\
\hline & Rocker panel, etc. & Small & Simple & $\begin{array}{c}\text { Avail- } \\
\text { able }\end{array}$ & Small & Large \\
\hline
\end{tabular}

\begin{tabular}{||c|c|}
\hline Injection & $\begin{array}{c}\text { Productivity } \\
\text { (molding cycle) }\end{array}$ \\
\hline Compression & $\begin{array}{c}\text { Facility cost } \\
\text { (press pressure) }\end{array}$ \\
\hline Compression & $\begin{array}{c}\text { Facility cost } \\
\text { (press pressure) }\end{array}$ \\
\hline $\begin{array}{c}\text { Compression } \\
\text { Injection }\end{array}$ & $\begin{array}{c}\text { Facility cost } \\
\text { (press pressure) } \\
\text { Productivity } \\
\text { (molding cycle) }\end{array}$ \\
\hline $\begin{array}{c}\text { Compression } \\
\text { Facility cost (press } \\
\text { pressure) }\end{array}$ \\
\hline $\begin{array}{c}\text { Facility cost } \\
\text { Injection }\end{array}$ & $\begin{array}{c}\text { (press pressure) } \\
\text { Productivity } \\
\text { (molding cycle) }\end{array}$ \\
\hline Compression & $\begin{array}{c}\text { Facility cost } \\
\text { (press pressure) }\end{array}$ \\
\hline Compression & $\begin{array}{c}\text { Facility cost } \\
\text { (press pressure) }\end{array}$ \\
\hline Compression & - \\
\hline Compression & - \\
\hline
\end{tabular}


accommodate the need for future conversion to CFRP.

We first considered the floor pan, a body panel of significant dimensions. Since this panel is characterized by complex concaves and protrusions in the tunnel sections, an evaluation based on the results described above pointed to the injection method as being more suitable than the compression method. On the other hand, sub-frames and side sills, which are frame elements, have medium-scale dimensions and are relatively simple in shape. For these reasons, evaluation pointed to the compression method being more suitable. However, the injection method would have to be developed for mass production, while a press facility with a capacity of at least 1000 tons would be needed for the compression method. There are still issues with mass production, however, which will be a requirement in the future. Lids such as engine hoods and tailgates were considered in terms of their being bonded structures with inner and outer panels. Such components have medium-scale dimensions but attention to design is particularly important for the outer panel. Demands for shapes range from quite simple ones to complex ones such as bulges on engine hoods or spoiler-integrated types for tailgates. It would appear that the molding method must be decided according to the complexity of such shapes. Even in the case of lids, for instance, they are not all the same and the required physical properties vary from one to another. Tailgates, for instance, require a certain degree of impact resistance to be able to withstand rear-end collisions, while the impact resistance of engine hoods cannot be increased due to the necessity to provide pedestrian protection. Another aspect that should be noted with regards to the design is the sense of unity for the entire vehicle. If the outer panels were to be produced using different molding methods, then a difference in the surface quality would arise. As a result, the likes of the paint coating that is performed as a post-process would also need to be changed. Thus, the manufacturing issue shifts from the molding method to the paint finishing. Therefore, the method used to mold lids must be selected at the stage where the product is being commercialized. In any event, there is no doubt that issues related to the molding method, which are similar to those for the body frame, will also arise in such instances.

Reinforcement components such as engine mounts, or interior and exterior components such as rear spoilers and rocker panels, tend to be well suited to the application of the compression method due to their required characteristics and, as such, fundamental problems should not arise.

It is possible to evaluate whether the injection or compression method is compatible with the main components of an automobile body, although there are some for which it was difficult to make a selection, such as the lids described above. Both molding methods incur significant issues that must be resolved to accommodate the need for mass production in the future.

\section{Discussions Relating to CFRP Molding Methods for Automobile Bodies}

In the future, it will be vital to identify the direction in which improvements will have to be made, by addressing trends in the technologies that have already been developed, in relation to the problems related to the two molding methods described above. 
Fig.2, below plots the fundamental problems with the injection method on the horizontal axis, while the pressure applied by the press, which is the main issue with the compression method, is plotted on the vertical axis. On this graph, we have plotted representative examples that have been developed to date.

More than 30 years ago, in 1980, the "Research and Development for Next Generation Composite Materials" project was started as a national effort, which brought up similar discussions. RTM, which is an injection method, was referred to as "temperature-regulated RTM" and attempts were made to develop a technology to significantly reduce the molding cycle. On the other hand, research was conducted to develop technology for reducing the pressure exerted by the press, which came to be known as the "stretch and press combined method" for SMC, which is a compression method. The relevant targets are also indicated in the diagram and while a considerable number of successes were obtained, significant failures were also confirmed ${ }^{2}$. Since then, the New Energy and Industrial Technology Development Organization (NEDO) has been established and rapid-curing resins have been developed for the

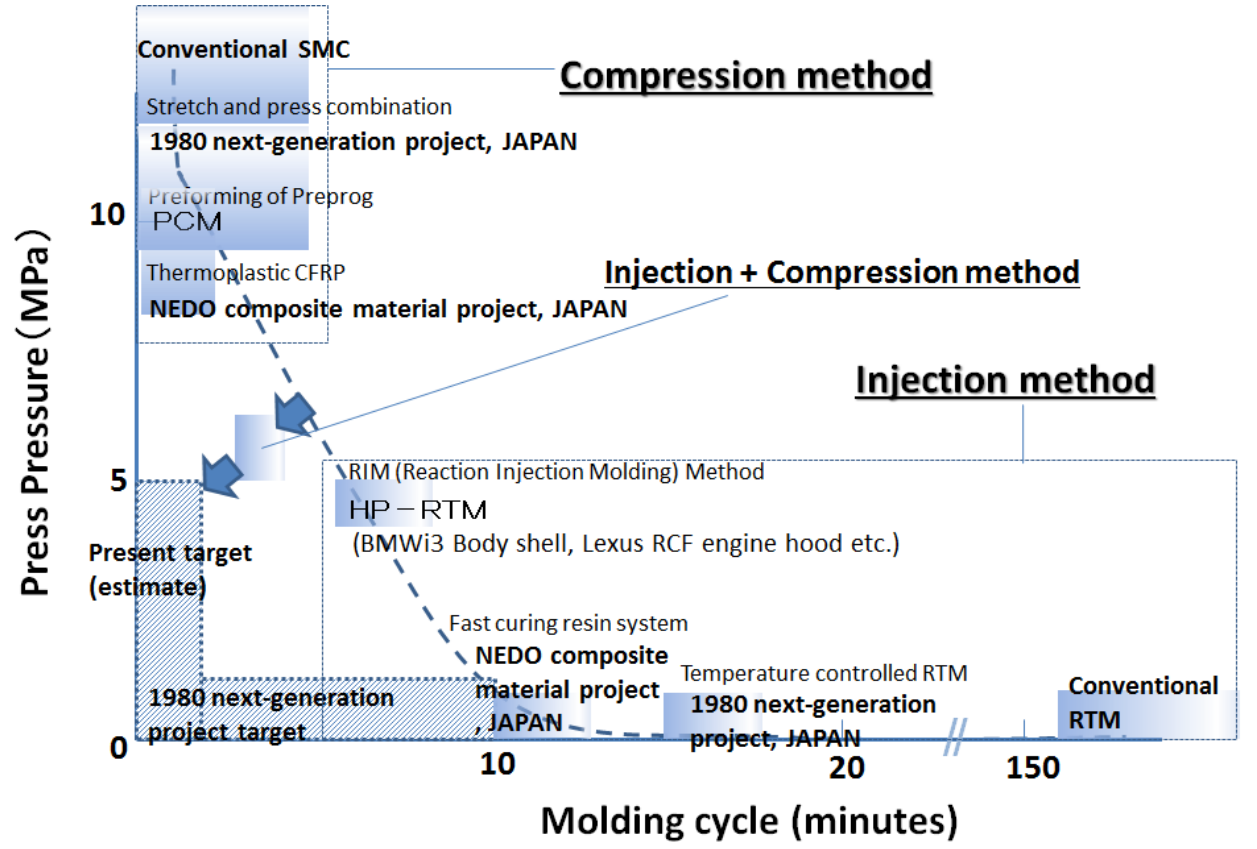

Fig.2. Transition of research and development on issues

while thermoplastic-resin CFRP has been researched for application to the compression method. This study is ongoing, with the ultimate goal of developing a thermoplastic-resin CFRP. The figure shows that the targets set in 1980 have been partially achieved. The targets, however, have changed as the expectations for CFRP grew, with molding cycles in the order of one minute now expected, which is significantly less than what was initially expected. Conversely, the trend is toward less demanding press capacities. A general purpose press will often have a capacity in the order of 
$500 \mathrm{MPa}$, as plotted in the figure. Further margins for improvement have emerged in this area, however.

HP-RTM has been adopted as the injection method for the body of the BMW i3 as well as the engine hood and roof of the Lexus RCF, as described above, while PCM was adopted as the compression method for the trunk lid of the Nissan GT-R. In every one of these cases, however, the plotted targets have not been satisfied. A multiple method involving both injection and compression molding to take advantage of the respective characteristics of each method has also been considered, but more technology implementations will be necessary to make that a reality. It would be necessary to conduct discussions on the validity of plotted targets, as well as perform a thorough investigation on the development direction that must be adopted in the future. Moreover, because materials of the main parts changing greatly influences not only the molding process but also the specifications of post-processes such as assembly and painting, the appearance of CFRP parts might be a good chance to review the way of the automobile manufacturing method in the future. It is very meaningful that a variety of opinion of automobile companies are aggregated and analyzed by management engineering,etc.

\section{Conclusion}

CFRP molding methods for automobile bodies can be largely categorized into injection and compression methods. While the two methods differ in many aspects, the essential difference between the two lies in whether the process involves an intermediary material. This had a significant impact on quality and productivity. An evaluation was performed to determine which of these methods was most appropriate for basic molding, by comparing the nature of the intermediary material with the main components of an automobile body, which led to the discovery of fundamental issues. A review of technical developments attained up to this point, considering these fundamental issues, was undertaken. As a result, we found that, while significant progress had been made, there was still a divergence from the expected target. It became clear that further development and control of the resin employed for the CFRP was important to establishing a direction to take to attain improvements. In the future, our aim is to gain an understanding of the validity of the established targets, perform quantification of the fluidity targets for the resin by simulation, and achieve further improvements in the technical aspects through enhanced collaboration with resin manufacturers. We also intend to make plans for implementing analyses and discussions not only relating to the technical aspects but also to business management.

\section{References}

1. Yuji Kageyama: " Today's and Future's CFRP Technologies for Automobile (Special Issue on Increasing Enhancements and Applications of Carbon Fiber Plastics in Automobiles)", Journal of Society of Automotive Engineers of Japan, Vol. 68, No. 11, 2014, pp. 75-81. http://ci.nii.ac.jp/naid/40020256901

2. Japanese Standards Association: Next Generation Composite Material Technology Handbook, 1990. 